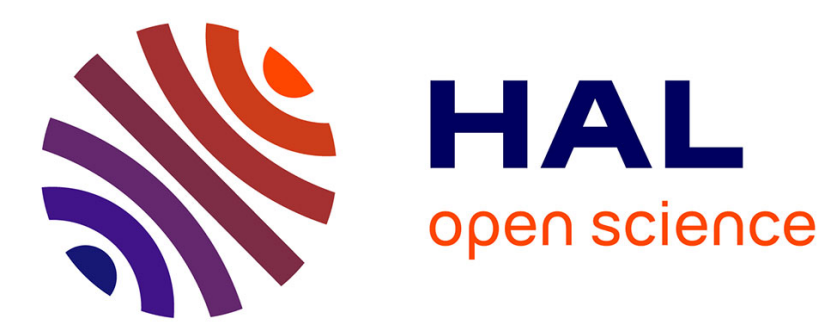

\title{
Asymptotic behavior of oscillatory fractional processes
}

Renaud Marty, Knut Solna

\section{To cite this version:}

Renaud Marty, Knut Solna. Asymptotic behavior of oscillatory fractional processes. Lecture Notes in Mathematics, 2012, Séminaire de Probabilités XLIV, 2046, pp.247-269. hal-00558967

\section{HAL Id: hal-00558967 https://hal.science/hal-00558967}

Submitted on 24 Jan 2011

HAL is a multi-disciplinary open access archive for the deposit and dissemination of scientific research documents, whether they are published or not. The documents may come from teaching and research institutions in France or abroad, or from public or private research centers.
L'archive ouverte pluridisciplinaire HAL, est destinée au dépôt et à la diffusion de documents scientifiques de niveau recherche, publiés ou non, émanant des établissements d'enseignement et de recherche français ou étrangers, des laboratoires publics ou privés. 


\title{
Asymptotic behavior of oscillatory fractional processes
}

\author{
Renaud Marty*and Knut Sølna ${ }^{\dagger}$
}

Key-words: Fractional processes, Brownian motion, waves in random media.

AMS classification (2000): 60F17, 60G10, 60G15.

\begin{abstract}
In this paper we consider the antiderivative of the product of a fractional random process and a periodic function. We establish that the rescaled process constructed in this way converges to a Brownian motion whose variance depends on the frequency of the periodic function and the Hurst parameter. We also prove that for two different frequencies the limits are independent. Finally, we discuss applications to wave propagation in random media.
\end{abstract}

\section{Introduction}

Fractional processes have attracted a lot of attention in recent years. They provide relevant models for long-range dependence or antipersistent random behavior [11].

Fractional Brownian motion is the most famous fractional process. It satisfies many relevant properties such as self-similarity, Gaussianity and stationarity of the increments.

Another crucial property for applications is the invariance principle [11]. Let $H \in(0,1), B_{H}=\left\{B_{H}(z)\right\}_{z}$ be a fractional Brownian motion with Hurst index $H$. Depending on the value of $H$, the fractional white noise

$$
\delta B_{H}=\left\{B_{H}(z+1)-B_{H}(z)\right\}_{z}
$$

(which is stationary) satisfies various "memory-properties". For $H \in(1 / 2,1)$ the process $\delta B_{H}$ is long-range dependent, for $H=1 / 2$ it is mixing, and for $H \in(0,1 / 2)$ it is antipersistent. Let consider a stationary and Gaussian process $\nu=\{\nu(z)\}_{z}$ that satisfies roughly the same range property as the fractional white noise $\delta B_{H}$, that is, it has the same decay rate of the integrated correlation. Then, the invariance principle says that when $\varepsilon$ goes to 0 , the rescaled process

$$
\left\{\varepsilon^{2 H} \int_{0}^{z / \varepsilon^{2}} \nu(y) d y\right\}_{z}=\left\{\frac{1}{\varepsilon^{2-2 H}} \int_{0}^{z} \nu\left(\frac{y}{\varepsilon^{2}}\right) d y\right\}_{z}
$$

\footnotetext{
*Institut Elie Cartan de Nancy, Nancy-Université, CNRS, INRIA, B.P. 239, F-54506 Vandoeuvre-lès-Nancy Cedex, France. renaud.marty@iecn.u-nancy.fr

${ }^{\dagger}$ University of California at Irvine, Irvine, CA 92697-3875, USA. ksolna@math.uci.edu
} 
converges in distribution to the fractional Brownian motion $B_{H}$. Notice that the invariance principle is of great importance. In particular it makes the increments of $B_{H}$ serve as universal stationary Gaussian models with various memory properties depending on the index $H$ [11].

Applications of fractional processes to wave propagation in random media have recently been studied in $[5,9,10]$. Here they are used to model very complex and long range media. In addition to random phenomena, due to the nature of waves, periodic modulation arises naturally in harmonic descriptions associated with wave phenomena, [2]. This then leads to the study of evolution problems driven by periodic and random processes. A simple example is the asymptotic study of the process defined by

$$
v_{p}^{\varepsilon}=\left\{\frac{1}{\varepsilon^{\gamma}} \int_{0}^{z} \nu\left(\frac{y}{\varepsilon^{2}}\right) p\left(\frac{y}{\varepsilon^{\tau}}\right) d y\right\}_{z}
$$

where $\tau$ and $\gamma$ are two positive constants and $p$ is a periodic function. Such a question have been investigated in particular cases in [8]. It was proven that if $p=\cos (\omega \times \cdot)($ or $\sin (\omega \times \cdot)), \tau \in(0,2), \gamma=1+(2-\tau)(1 / 2-H)$ and $\nu$ is a Gaussian and stationary process that is essentially a fractional white noise, then $v_{p}^{\varepsilon}$ converges in distribution to a Brownian motion. The result with $H=1 / 2$ had been known for many years and applied to wave propagation in mixing media, see [2], while the results with $H \in(0,1)$ allowed for the study of waves in fractional media.

In the present paper we aim to generalize the result presented above. Our framework is based on a Gaussian process $\nu$ defined in terms of a general harmonizable representation. We first consider functions $p=\cos (\omega \times \cdot)$ (or $\sin (\omega \times \cdot)$ ) and $\tau \in(0,2)$. The proof of the convergence of $v_{p}^{\varepsilon}$ in [8] was based on the particular form of the covariance of $\nu$ and the cases $H<$ $1 / 2, H=1 / 2$ or $H>1 / 2$ had to be considered separately. Here we give a new proof that unifies the three cases for $H$ and that is based on the harmonizable representation and thus more general. We also consider general period functions $p$. We prove that under general assumptions on $p$ the process converges to a Brownian motion whose covariance depends on the Fourier coefficients of $p$.

A second point we address is the correlation of the limits for different frequencies. Let $\omega_{1}$ and $\omega_{2}$ be two real numbers satisfying $\omega_{1} \neq \omega_{2}$ and $\omega_{1} \neq-\omega_{2}$. It is well known in the mixing case $(H=1 / 2)$, that if we denote respectively by $B^{\omega_{1}}$ and $B^{\omega_{2}}$ the limits of $v_{p}^{\varepsilon}$ with $p=\cos \left(\omega_{1} \times \cdot\right)$ and $p=\cos \left(\omega_{2} \times \cdot\right)$ respectively, then the limits $B^{\omega_{1}}$ and $B^{\omega_{2}}$ are independent [2]. This asymptotic decorrelation is a crucial property in the applications to waves. Here we generalize this result to every $H \in(0,1)$.

The assumption $\tau \in(0,2)$ corresponds to models for wave propagations where the phases are slowly varying with respect to the random fluctuations [2]. Another problem of interest is when phases oscillate at the same speed as the random medium fluctuations. Physically this corresponds to a strong "resonance" between the waves and the fluctuations. This motivates studying the case $\tau=2$ and then, in the mixing case, one obtains different results from those of the case $\tau \in(0,2)$. In particular, the effective second moment coefficient can be written as $\int_{0}^{\infty} \mathbb{E}[\nu(0) \nu(y)] d y$ if $\tau \in(0,2)$ and $\int_{0}^{\infty} \cos (\omega y) \mathbb{E}[\nu(0) \nu(y)] d y$ if $\tau=2[2]$. In the present paper we analyze the specific behavior when $\tau=2$ for the full range of $H \in(0,1)$. 
In conclusion, we discuss some possible extensions of our work for applications to wave propagation. In particular we give a conjecture that would generalize the study of wave propagation in mixing [1, 2] or long-range [9] media to a general fractional media.

Our paper is organized as follows. In Section 2 we establish the main results and we prove them in Section 3. In Section 4 we extend our results to general periodic functions. In Section 5 we discuss applications to the study of wave propagation in random media.

\section{Assumptions and main result}

\subsection{Assumptions}

Here we describe the process that we will be working with throughout the paper. We define $\nu$ for every $z \geq 0$ by

$$
\nu(z)=\int_{\mathbb{R}} \exp (i z \zeta) \psi(\zeta)|\zeta|^{1 / 2-H} \widehat{W}(d \zeta)
$$

where $H \in(0,1)$ and $\psi$ is a complex-valued function and $\widehat{W}(d \zeta)$ is the Fourier transform of a real Gaussian measure. We assume that $\psi$ is continuous and even and satisfies $|\psi(\zeta)|=\mathcal{O}_{|\zeta| \rightarrow \infty}\left(|\zeta|^{-1}\right)$. Thus $\nu$ is a centered and stationary Gaussian process and its covariance takes the form

$$
r(z)=\mathbb{E}[\nu(0) \nu(z)]=\int_{\mathbb{R}} \exp (i z \zeta)|\psi(\zeta)|^{2}|\zeta|^{1-2 H} d \zeta .
$$

An integration with respect to $z$ gives

$$
\int_{0}^{Z} r(z) d z \sim \sigma(H) Z^{2 H-1}
$$

as $Z \rightarrow \infty$ with

$$
\sigma(H)=2|\psi(0)|^{2} \int_{0}^{\infty} \frac{\sin (\zeta)}{\zeta^{2 H}} d \zeta .
$$

We deduce from (4), depending on the value of $H$, that the process $\nu$ satisfies one of the three following properties: $\int_{0}^{\infty} r(z) d z=\infty$ if $H \in(1 / 2,1)$, $\int_{0}^{\infty} r(z) d z \in(0, \infty)$ if $H=1 / 2$ and $\int_{0}^{\infty} r(z) d z=0$ if $H \in(0,1 / 2)$. In other words $\nu$ is long-range dependent when $H>1 / 2$, antipersistent when $H<1 / 2$ and satisfies a general mixing property when $H=1 / 2$.

We conclude this subsection by two examples of processes satisfying the assumptions cited above. These examples are defined in terms of the (nonstandard) fractional Brownian motion defined for every $z$ by

$$
B_{H}(z)=\frac{1}{\rho(H)} \int_{\mathbb{R}} \frac{e^{i z \zeta}-1}{i \zeta|\zeta|^{H-1 / 2}} \widehat{W}(d \zeta)
$$

where $\rho(H)$ is a renormalization constant defined by

$$
\rho(H)^{2}=\int_{\mathbb{R}} \frac{\left|e^{i \zeta}-1\right|^{2}}{|\zeta|^{2 H+1}} d \zeta
$$


The first example we present is the fractional white noise $\delta B_{H}$ defined as

$$
\delta B_{H}(z)=B_{H}(z+1)-B_{H}(z)=\frac{1}{\rho(H)} \int_{\mathbb{R}} \exp (i z \zeta) \frac{e^{i \zeta}-1}{i \zeta|\zeta|^{H-1 / 2}} \widehat{W}(d \zeta) .
$$

In this example

$$
\psi(\zeta)=\frac{e^{i \zeta}-1}{\rho(H) i \zeta} .
$$

The second example is the fractional Ornstein-Uhlenbeck (OU) process. Recall that the stationary fractional OU process $X_{H}$ can be written in terms of a the fractional Brownian motion $B_{H}$ as

$$
\begin{aligned}
X_{H}(z) & =B_{H}(z)-e^{-z} \int_{-\infty}^{z} e^{y} B_{H}(y) d y \\
& =\frac{1}{\rho(H)} \int_{\mathbb{R}} \frac{\exp (i z \zeta)}{1+i \zeta} \frac{\widehat{W}(d \zeta)}{|\zeta|^{H-1 / 2}}
\end{aligned}
$$

so the corresponding function $\psi$ is

$$
\psi(\zeta)=\frac{1}{\rho(H)(1+i \zeta)} .
$$

\subsection{Main result}

Here we consider a general process $\nu$ as defined in the previous subsection and throughout the paper we assume that $H \in(0,1)$. We introduce the two processes $v_{c}^{\omega, \varepsilon}$ and $v_{s}^{\omega, \varepsilon}$ defined by

$$
v_{c}^{\omega, \varepsilon}(z)=\frac{1}{\varepsilon^{\gamma}} \int_{0}^{z} \nu\left(\frac{y}{\varepsilon^{2}}\right) \cos \left(\omega \frac{y}{\varepsilon^{\tau}}\right) d y
$$

and

$$
v_{s}^{\omega, \varepsilon}(z)=\frac{1}{\varepsilon^{\gamma}} \int_{0}^{z} \nu\left(\frac{y}{\varepsilon^{2}}\right) \sin \left(\omega \frac{y}{\varepsilon^{\tau}}\right) d y
$$

with $\nu$ defined as in (2), $\omega \neq 0$, while $\gamma$ and $\tau$ are constants and $\varepsilon$ is a small parameter. We assume that $\tau \in(0,2]$ and $\gamma$ is given by

$$
\gamma=1+(2-\tau)(1 / 2-H) .
$$

Notice that in the particular case $\tau=2$ we have $\gamma=1$.

We fix an $n$-dimensional vector of frequencies $\Omega=\left(\omega_{1}, \ldots, \omega_{n}\right)$ such that $\omega_{j} \neq 0$ for every $j$ and $\omega_{j} \neq \omega_{k}$ and $\omega_{j} \neq-\omega_{k}$ for every $j \neq k$. We define the $2 n$-dimensional process $V^{\Omega, \varepsilon}$ by

$$
V^{\Omega, \varepsilon}=\left(v_{c}^{\omega_{1}, \varepsilon}, v_{s}^{\omega_{1}, \varepsilon}, v_{c}^{\omega_{2}, \varepsilon}, v_{s}^{\omega_{2}, \varepsilon}, \cdots, v_{c}^{\omega_{n}, \varepsilon}, v_{s}^{\omega_{n}, \varepsilon}\right) .
$$

We state the first main theorem of this paper.

Theorem 1. The finite dimensional distributions of the process $V^{\Omega, \varepsilon}$ converge to those of a $2 n$-dimensional Brownian motion $B^{\Omega}$ defined by

$$
B^{\Omega}=\left(B_{c}^{\omega_{1}}, B_{s}^{\omega_{1}}, B_{c}^{\omega_{2}}, B_{s}^{\omega_{2}}, \cdots, B_{c}^{\omega_{n}}, B_{s}^{\omega_{n}}\right)
$$


whose coordinates are independent and satisfy

$$
\mathbb{E}\left[\left(B_{c}^{\omega_{j}}(1)\right)^{2}\right]=\mathbb{E}\left[\left(B_{s}^{\omega_{j}}(1)\right)^{2}\right]=C\left(\omega_{j}\right)^{2}
$$

for every $j$ and where the second moment coefficient

$$
C(\omega)^{2}=\frac{\widetilde{\psi}(\omega)}{2} \int_{-\infty}^{\infty} \frac{\left|e^{i \zeta}-1\right|^{2}}{|\zeta|^{2}} d \zeta=\pi \widetilde{\psi}(\omega)
$$

with $\widetilde{\psi}(\omega)=|\psi(0)|^{2}|\omega|^{1-2 H}$ if $\tau \in(0,2)$ and $\widetilde{\psi}(\omega)=|\psi(\omega)|^{2}|\omega|^{1-2 H}$ if $\tau=2$.

Notice that the cases $\tau \in(0,2)$ (the slow-phase case) and $\tau=2$ (the fast-phase case) are dramatically different. The second moment depend on the whole function $\psi$ if $\tau=2$ while it does not if $\tau \in(0,2)$. When $\tau=2$ the analogue in the wave case is that the wave resolves the full spectrum of the fluctuations. This theorem generalizes the diffusion-approximation theory set forth in [2]. Indeed, if we consider the case $H=1 / 2$, we have

$$
\begin{aligned}
\int_{0}^{A} r(z) \cos (\omega z) d z= & \int_{0}^{A} \cos (\omega z) \int_{\mathbb{R}} \exp (i z \zeta)|\psi(\zeta)|^{2} d \zeta d z \\
= & \frac{1}{2} \int_{\mathbb{R}}|\psi(\zeta / A+\omega)|^{2} \frac{e^{i \zeta}-1}{i \zeta} d \zeta \\
& +\frac{1}{2} \int_{\mathbb{R}}|\psi(\zeta / A-\omega)|^{2} \frac{e^{i \zeta}-1}{i \zeta} d \zeta .
\end{aligned}
$$

Then, because $\psi$ is continuous and even we get

$$
\int_{0}^{\infty} r(z) \cos (\omega z) d z=|\psi(\omega)|^{2} \int_{\mathbb{R}} \frac{\sin (\zeta)}{\zeta} d \zeta=\pi|\psi(\omega)|^{2}
$$

It then follows that the constant $C(\omega)$ (depending on the value of $\tau$ ) corresponds to the effective coefficients found in the diffusion-approximation presented in [2].

To conclude this section we make a remark about the super-fast case that corresponds to $\tau>2$. We can observe, following the lines of the proof of the theorem, that the process $V^{\Omega, \varepsilon}$ converges to $0_{\mathbb{R}^{2 n}}$ as already observed in the classical approximation-diffusion theory (for $H=1 / 2$, see for instance [3]).

\section{Proof of Theorem 1}

In the first part of the proof we introduce some notation. Next we establish that, for a fixed frequency $\omega$, the 2-dimensional process $\left(v_{c}^{\omega, \varepsilon}, v_{s}^{\omega, \varepsilon}\right)$ defined by (5) and (6) converges in the sense of the finite dimensional distributions to the 2-dimensional Brownian motion $\left(B_{c}^{\omega}, B_{s}^{\omega}\right)$ defined by (9). In the third part we prove that for two different frequencies $\omega_{1}$ and $\omega_{2}$ the limits $\left(B_{c}^{\omega_{1}}, B_{s}^{\omega_{1}}\right)$ and $\left(B_{c}^{\omega_{2}}, B_{s}^{\omega_{2}}\right)$ are independent. In the proof we use technical lemmas whose derivations are postponed to the end of this section. 


\subsection{Preliminaries}

We introduce the complex-variable function $g$ defined for every $u \in \mathbb{C}$ by

$$
g(u)=\frac{\exp (u)-1}{u} .
$$

By a direct integration we obtain

$$
\begin{aligned}
\int_{0}^{z} \exp \left(\frac{i y \zeta}{\varepsilon^{2}}\right) & \cos \left(\omega \frac{y}{\varepsilon^{\tau}}\right) d y \\
= & \frac{z}{2}\left\{g\left(i z\left(\frac{\zeta}{\varepsilon^{2}}-\frac{\omega}{\varepsilon^{\tau}}\right)\right)+g\left(i z\left(\frac{\zeta}{\varepsilon^{2}}+\frac{\omega}{\varepsilon^{\tau}}\right)\right)\right\}
\end{aligned}
$$

and

$$
\begin{aligned}
\int_{0}^{z} \exp \left(\frac{i y \zeta}{\varepsilon^{2}}\right) & \sin \left(\omega \frac{y}{\varepsilon^{\tau}}\right) d y \\
= & \frac{i z}{2}\left\{g\left(i z\left(\frac{\zeta}{\varepsilon^{2}}-\frac{\omega}{\varepsilon^{\tau}}\right)\right)-g\left(i z\left(\frac{\zeta}{\varepsilon^{2}}+\frac{\omega}{\varepsilon^{\tau}}\right)\right)\right\}
\end{aligned}
$$

Then we can deduce that for every $\omega, \varepsilon$ and $z$ we have

$$
v_{c}^{\omega, \varepsilon}(z)=\int_{\mathbb{R}} g_{c}^{\omega, \varepsilon}(z, \zeta) \widehat{W}(d \zeta)
$$

and

$$
v_{s}^{\omega, \varepsilon}(z)=\int_{\mathbb{R}} g_{s}^{\omega, \varepsilon}(z, \zeta) \widehat{W}(d \zeta)
$$

where

$$
g_{c}^{\omega, \varepsilon}(z, \zeta)=\frac{z}{2 \varepsilon^{\gamma}} \frac{\psi(\zeta)}{|\zeta|^{H-1 / 2}}\left\{g\left(i z\left(\frac{\zeta}{\varepsilon^{2}}-\frac{\omega}{\varepsilon^{\tau}}\right)\right)+g\left(i z\left(\frac{\zeta}{\varepsilon^{2}}+\frac{\omega}{\varepsilon^{\tau}}\right)\right)\right\}
$$

and

$$
g_{s}^{\omega, \varepsilon}(z, \zeta)=\frac{i z}{2 \varepsilon^{\gamma}} \frac{\psi(\zeta)}{|\zeta|^{H-1 / 2}}\left\{g\left(i z\left(\frac{\zeta}{\varepsilon^{2}}-\frac{\omega}{\varepsilon^{\tau}}\right)\right)-g\left(i z\left(\frac{\zeta}{\varepsilon^{2}}+\frac{\omega}{\varepsilon^{\tau}}\right)\right)\right\} .
$$

It is convenient to introduce the integral

$$
\begin{aligned}
& I_{1}^{\varepsilon}\left(\omega_{1}, \omega_{2}, z_{1}, z_{2}\right) \\
& \quad=\frac{z_{1} z_{2}}{4 \varepsilon^{2 \gamma}} \int_{\mathbb{R}} \frac{|\psi(\zeta)|^{2}}{|\zeta|^{2 H-1}} g\left(i z_{1}\left(\frac{\zeta}{\varepsilon^{2}}-\frac{\omega_{1}}{\varepsilon^{\tau}}\right)\right) g\left(-i z_{2}\left(\frac{\zeta}{\varepsilon^{2}}-\frac{\omega_{2}}{\varepsilon^{\tau}}\right)\right) d \zeta
\end{aligned}
$$

and

$$
\begin{aligned}
I_{2}^{\varepsilon}\left(\omega_{1}, \omega_{2}, z_{1}, z_{2}\right) & =I_{1}^{\varepsilon}\left(\omega_{1},-\omega_{2}, z_{1}, z_{2}\right), \\
I_{3}^{\varepsilon}\left(\omega_{1}, \omega_{2}, z_{1}, z_{2}\right) & =I_{1}^{\varepsilon}\left(-\omega_{1}, \omega_{2}, z_{1}, z_{2}\right), \\
I_{4}^{\varepsilon}\left(\omega_{1}, \omega_{2}, z_{1}, z_{2}\right) & =I_{1}^{\varepsilon}\left(-\omega_{1},-\omega_{2}, z_{1}, z_{2}\right) .
\end{aligned}
$$




\subsection{Convergence at a fixed frequency}

Now we can state the convergence of $\left(v_{c}^{\omega, \varepsilon}, v_{s}^{\omega, \varepsilon}\right)$.

Lemma 1. The finite dimensional distributions of $\left(v_{c}^{\omega, \varepsilon}, v_{s}^{\omega, \varepsilon}\right)$ converge to those of $\left(B_{c}^{\omega}, B_{s}^{\omega}\right)$.

Proof. We first consider $v_{c}^{\omega, \varepsilon}$. For every $z_{1}$ and $z_{2}$ we have

$$
\begin{aligned}
\mathbb{E}\left[v_{c}^{\omega, \varepsilon}\left(z_{1}\right) v_{c}^{\omega, \varepsilon}\left(z_{2}\right)\right] & =\int_{\mathbb{R}} g_{c}^{\omega, \varepsilon}\left(z_{1}, \zeta\right) \bar{g}_{c}^{\omega, \varepsilon}\left(z_{2}, \zeta\right) d \zeta \\
& =\sum_{j=1}^{4} I_{j}^{\varepsilon}\left(\omega, \omega, z_{1}, z_{2}\right) .
\end{aligned}
$$

By the substitution $\zeta \rightarrow \varepsilon^{2} \zeta+\omega \varepsilon^{2-\tau}$ we can write

$$
I_{1}^{\varepsilon}\left(\omega, \omega, z_{1}, z_{2}\right)=\frac{z_{1} z_{2}}{4} \int_{\mathbb{R}} \frac{\left|\psi\left(\varepsilon^{2} \zeta+\omega \varepsilon^{2-\tau}\right)\right|^{2}}{\left|\varepsilon^{\tau} \zeta+\omega\right|^{2 H-1}} g\left(i z_{1} \zeta\right) g\left(-i z_{2} \zeta\right) d \zeta
$$

where we use $\gamma=1+(2-\tau)(1 / 2-H)$. It now follows from Lemma 3 below that we have the limit

$$
\begin{array}{r}
\lim _{\varepsilon \rightarrow 0} \int_{\mathbb{R}} \frac{\left|\psi\left(\varepsilon^{2} \zeta+\omega \varepsilon^{2-\tau}\right)\right|^{2}}{\left|\varepsilon^{\tau} \zeta+\omega\right|^{2 H-1}} g\left(i z_{1} \zeta\right) g\left(-i z_{2} \zeta\right) d \zeta \\
=\widetilde{\psi}(\omega) \int_{\mathbb{R}} g\left(i z_{1} \zeta\right) g\left(-i z_{2} \zeta\right) d \zeta
\end{array}
$$

where $\widetilde{\psi}(\omega)=|\psi(0)|^{2}|\omega|^{1-2 H}$ if $\tau \in(0,2)$ and $\widetilde{\psi}(\omega)=|\psi(\omega)|^{2}|\omega|^{1-2 H}$ if $\tau=2$. We deal with the integral $I_{4}^{\varepsilon}\left(\omega, \omega, z_{1}, z_{2}\right)$ in the same way so that we get

$$
\begin{aligned}
\lim _{\varepsilon \rightarrow 0}\left(I_{1}^{\varepsilon}\left(\omega, \omega, z_{1}, z_{2}\right)+I_{4}^{\varepsilon}\left(\omega, \omega, z_{1}, z_{2}\right)\right) \\
=\frac{z_{1} z_{2} \widetilde{\psi}(\omega)}{2} \int_{\mathbb{R}} g\left(i z_{1} \zeta\right) g\left(-i z_{2} \zeta\right) d \zeta .
\end{aligned}
$$

Now we deal with $I_{2}^{\varepsilon}\left(\omega, \omega, z_{1}, z_{2}\right)$. By the same substitution as for $I_{1}^{\varepsilon}\left(\omega, \omega, z_{1}, z_{2}\right)$ we have

$$
\begin{aligned}
& I_{2}^{\varepsilon}\left(\omega, \omega, z_{1}, z_{2}\right) \\
& \quad=\frac{z_{1} z_{2}}{4} \int_{\mathbb{R}} \frac{\left|\psi\left(\varepsilon^{2} \zeta+\omega \varepsilon^{2-\tau}\right)\right|^{2}}{\left|\varepsilon^{\tau} \zeta+\omega\right|^{2 H-1}} g\left(i z_{1} \zeta\right) g\left(-i z_{2}\left(\zeta+2 \omega \varepsilon^{-\tau}\right)\right) d \zeta .
\end{aligned}
$$

It is now enough to prove that the last integral converges to 0 and this is due to Lemma 4 below. We deal with the integral $I_{3}^{\varepsilon}\left(\omega, \omega, z_{1}, z_{2}\right)$ in the same way so that we obtain again by Lemma 4

$$
\lim _{\varepsilon \rightarrow 0}\left(I_{2}^{\varepsilon}\left(\omega, \omega, z_{1}, z_{2}\right)+I_{3}^{\varepsilon}\left(\omega, \omega, z_{1}, z_{2}\right)\right)=0
$$

and thus, using (13),

$$
\lim _{\varepsilon \rightarrow 0} \mathbb{E}\left[v_{c}^{\omega, \varepsilon}\left(z_{1}\right) v_{c}^{\omega, \varepsilon}\left(z_{2}\right)\right]=\frac{z_{1} z_{2} \widetilde{\psi}(\omega)}{2} \int_{\mathbb{R}} g\left(i z_{1} \zeta\right) g\left(-i z_{2} \zeta\right) d \zeta,
$$


which means that the finite dimensional distributions of $v_{c}^{\omega, \varepsilon}$ converge to those of the Brownian motion $B_{c}^{\omega}$ with the scaling (9). Using the same argument we also prove that the finite dimensional distribution of $v_{s}^{\omega, \varepsilon}$ converge to those of the Brownian motion $B_{s}^{\omega}$ with scaling (9). Now it remains to establish the independence between $B_{c}^{\omega}$ and $B_{s}^{\omega}$. We can write for every $z_{1}$ and $z_{2}$

$$
\begin{aligned}
\mathbb{E}\left[v_{c}^{\omega, \varepsilon}\left(z_{1}\right) v_{s}^{\omega, \varepsilon}\left(z_{2}\right)\right] & =\int_{\mathbb{R}} g_{c}^{\omega, \varepsilon}\left(z_{1}, \zeta\right) \bar{g}_{s}^{\omega, \varepsilon}\left(z_{2}, \zeta\right) d \zeta \\
& =i \sum_{j=1}^{4}(-1)^{j} I_{j}^{\varepsilon}\left(\omega, \omega, z_{1}, z_{2}\right) .
\end{aligned}
$$

From the asymptotic study of $I_{j}^{\varepsilon}\left(\omega, \omega, z_{1}, z_{2}\right)$ for $j \in\{1,2,3,4\}$ just above, we get

$$
\lim _{\varepsilon \rightarrow 0} \mathbb{E}\left[v_{c}^{\omega, \varepsilon}\left(z_{1}\right) v_{s}^{\omega, \varepsilon}\left(z_{2}\right)\right]=0
$$

which proves that $B_{c}^{\omega}$ and $B_{s}^{\omega}$ are independent and this concludes the proof.

\subsection{Asymptotic decorrelation for different frequencies}

Now we establish the asymptotic decorrelation between the vectors $\left(v_{c}^{\omega_{1}, \varepsilon}, v_{s}^{\omega_{1}, \varepsilon}\right)$ and $\left(v_{c}^{\omega_{2}, \varepsilon}, v_{s}^{\omega_{2}, \varepsilon}\right)$ when $\omega_{1} \neq \omega_{2}$ and $\omega_{1} \neq-\omega_{2}$.

Lemma 2. Let $z_{1}$ and $z_{2} \in \mathbb{R}$ and $\omega_{1}$ and $\omega_{2}$ such that $\omega_{1} \neq \omega_{2}$ and $\omega_{1} \neq-\omega_{2}$. We have

$$
\lim _{\varepsilon \rightarrow 0} \mathbb{E}\left[\left(\begin{array}{cc}
v_{c}^{\omega_{1}, \varepsilon}\left(z_{1}\right) v_{c}^{\omega_{2}, \varepsilon}\left(z_{2}\right) & v_{c}^{\omega_{1}, \varepsilon}\left(z_{1}\right) v_{s}^{\omega_{2}, \varepsilon}\left(z_{2}\right) \\
v_{s}^{\omega_{1}, \varepsilon}\left(z_{1}\right) v_{c}^{\omega_{2}, \varepsilon}\left(z_{2}\right) & v_{s}^{\omega_{1}, \varepsilon}\left(z_{1}\right) v_{s}^{\omega_{2}, \varepsilon}\left(z_{2}\right)
\end{array}\right)\right]=\left(\begin{array}{cc}
0 & 0 \\
0 & 0
\end{array}\right) .
$$

Proof. We consider $\omega_{1} \neq \omega_{2}$ and $\omega_{1} \neq-\omega_{2}$. To prove the lemma it is sufficient to establish the limit of $\mathbb{E}\left[v_{c}^{\omega_{1}, \varepsilon}\left(z_{1}\right) v_{c}^{\omega_{2}, \varepsilon}\left(z_{2}\right)\right]$ and $\mathbb{E}\left[v_{c}^{\omega_{1}, \varepsilon}\left(z_{1}\right) v_{s}^{\omega_{2}, \varepsilon}\left(z_{2}\right)\right]$. The asymptotic study of $\mathbb{E}\left[v_{s}^{\omega_{1}, \varepsilon}\left(z_{1}\right) v_{s}^{\omega_{2}, \varepsilon}\left(z_{2}\right)\right]$ and $\mathbb{E}\left[v_{s}^{\omega_{1}, \varepsilon}\left(z_{1}\right) v_{c}^{\omega_{2}, \varepsilon}\left(z_{2}\right)\right]$ respectively will be similar. Let us start by $\mathbb{E}\left[v_{c}^{\omega_{1}, \varepsilon}\left(z_{1}\right) v_{c}^{\omega_{2}, \varepsilon}\left(z_{2}\right)\right]$. We have

$$
\mathbb{E}\left[v_{c}^{\omega_{1}, \varepsilon}\left(z_{1}\right) v_{c}^{\omega_{2}, \varepsilon}\left(z_{2}\right)\right]=\sum_{j=1}^{4} I_{j}^{\varepsilon}\left(\omega_{1}, \omega_{2}, z_{1}, z_{2}\right)
$$

As in the case of $I_{2}^{\varepsilon}\left(\omega, \omega, z_{1}, z_{2}\right)$ in the proof of Lemma 1 we can write by the substitution $\zeta \rightarrow \varepsilon^{2} \zeta+\omega_{1} \varepsilon^{2-\tau}$

$$
\begin{aligned}
& I_{1}^{\varepsilon}\left(\omega_{1}, \omega_{2}, z_{1}, z_{2}\right) \\
& \quad=\frac{z_{1} z_{2}}{4} \int_{\mathbb{R}} \frac{\left|\psi\left(\varepsilon^{2} \zeta+\omega_{1} \varepsilon^{2-\tau}\right)\right|^{2}}{\left|\varepsilon^{\tau} \zeta+\omega_{1}\right|^{2 H-1}} g\left(i z_{1} \zeta\right) g\left(-i z_{2}\left(\zeta+\left(\omega_{1}-\omega_{2}\right) \varepsilon^{-\tau}\right)\right) d \zeta .
\end{aligned}
$$

Using Lemma 4 and the condition $\omega_{1} \neq \omega_{2}$ we get $\lim _{\varepsilon \rightarrow 0} I_{1}^{\varepsilon}\left(\omega_{1}, \omega_{2}, z_{1}, z_{2}\right)=$ 0 . Using the same approach and also either $\omega_{1} \neq \omega_{2}$ or $\omega_{1} \neq-\omega_{2}$ we obtain $\lim _{\varepsilon \rightarrow 0} I_{j}^{\varepsilon}\left(\omega_{1}, \omega_{2}, z_{1}, z_{2}\right)=0$ for $j=2,3$ and 4 also, thus

$$
\lim _{\varepsilon \rightarrow 0} \mathbb{E}\left[v_{c}^{\omega_{1}, \varepsilon}\left(z_{1}\right) v_{s}^{\omega_{2}, \varepsilon}\left(z_{2}\right)\right]=0 .
$$


It remains to study $\mathbb{E}\left[v_{c}^{\omega_{1}, \varepsilon}\left(z_{1}\right) v_{s}^{\omega_{2}, \varepsilon}\left(z_{2}\right)\right]$. We have

$$
\begin{aligned}
\mathbb{E}\left[v_{c}^{\omega_{1}, \varepsilon}\left(z_{1}\right) v_{s}^{\omega_{2}, \varepsilon}\left(z_{2}\right)\right] & =\int_{\mathbb{R}} g_{c}^{\omega_{1}, \varepsilon}\left(z_{1}, \zeta\right) \bar{g}_{s}^{\omega_{2}, \varepsilon}\left(z_{2}, \zeta\right) d \zeta \\
& =i \sum_{j=1}^{4}(-1)^{j} I_{j}^{\varepsilon}\left(\omega_{1}, \omega_{2}, z_{1}, z_{2}\right) .
\end{aligned}
$$

Using the same calculation as just above for $\mathbb{E}\left[v_{c}^{\omega_{1}, \varepsilon}\left(z_{1}\right) v_{c}^{\omega_{2}, \varepsilon}\left(z_{2}\right)\right]$ we get

$$
\lim _{\varepsilon \rightarrow 0} \mathbb{E}\left[v_{c}^{\omega_{1}, \varepsilon}\left(z_{1}\right) v_{s}^{\omega_{2}, \varepsilon}\left(z_{2}\right)\right]=0,
$$

which concludes the proof.

\subsection{Technical lemmas}

Lemma 3. We define $\widetilde{\psi}(\omega)=|\psi(0)|^{2}|\omega|^{1-2 H}$ if $\tau \in(0,2)$ and $\widetilde{\psi}(\omega)=$ $|\psi(\omega)|^{2}|\omega|^{1-2 H}$ if $\tau=2$. We have the following convergence

$$
\begin{array}{r}
\lim _{\varepsilon \rightarrow 0} \int_{\mathbb{R}} \frac{\left|\psi\left(\varepsilon^{2} \zeta+\omega \varepsilon^{2-\tau}\right)\right|^{2}}{\left|\varepsilon^{\tau} \zeta+\omega\right|^{2 H-1}} g\left(i z_{1} \zeta\right) g\left(-i z_{2} \zeta\right) d \zeta \\
=\widetilde{\psi}(\omega) \int_{\mathbb{R}} g\left(i z_{1} \zeta\right) g\left(-i z_{2} \zeta\right) d \zeta
\end{array}
$$

for every $z_{1}$ and $z_{2} \in \mathbb{R}$ and $\omega \neq 0$.

Proof. Without loss of generality and for simplicity of presentation we assume that $\omega>0$. We define for $a$ and $b$ in $[-\infty, \infty]$ the $\varepsilon$-dependent integral

$$
S^{\varepsilon}(a, b)=\int_{a}^{b} \frac{\left|\psi\left(\varepsilon^{2} \zeta+\omega \varepsilon^{2-\tau}\right)\right|^{2}}{\left|\varepsilon^{\tau} \zeta+\omega\right|^{2 H-1}} g\left(i z_{1} \zeta\right) g\left(-i z_{2} \zeta\right) d \zeta
$$

then, we need to study the convergence of $S^{\varepsilon}(-\infty, \infty)$. We fix $\eta>0$ sufficiently small and write

$$
S^{\varepsilon}(-\infty, \infty)=S^{\varepsilon}\left(-\infty,-\eta \varepsilon^{-\tau}\right)+S^{\varepsilon}\left(-\eta \varepsilon^{-\tau}, \eta \varepsilon^{-\tau}\right)+S^{\varepsilon}\left(\eta \varepsilon^{-\tau}, \infty\right) .
$$

By means of the substitution $\zeta \rightarrow \zeta / \varepsilon^{\tau}$ we get

$$
\begin{aligned}
& S^{\varepsilon}\left(-\infty,-\eta \varepsilon^{-\tau}\right) \\
& \quad=\varepsilon^{\tau} \int_{-\infty}^{-\eta} \frac{\left|\psi\left(\varepsilon^{2-\tau}(\zeta+\omega)\right)\right|^{2}}{z_{1} z_{2} \zeta^{2}|\zeta+\omega|^{2 H-1}}\left(e^{i z_{1} \zeta \varepsilon^{-\tau}}-1\right)\left(e^{-i z_{2} \zeta \varepsilon^{-\tau}}-1\right) d \zeta
\end{aligned}
$$

so that

$$
\left|S^{\varepsilon}\left(-\infty,-\eta \varepsilon^{-\tau}\right)\right| \leq \varepsilon^{\tau} \int_{-\infty}^{-\eta} \frac{\|\psi\|_{\infty}^{2}}{z_{1} z_{2} \zeta^{2}|\zeta+\omega|^{2 H-1}} d \zeta \rightarrow 0
$$

as $\varepsilon \rightarrow 0$. We deal with $S^{\varepsilon}\left(\eta \varepsilon^{-\tau}, \infty\right)$ in the same way and therefore it remains only to study $S^{\varepsilon}\left(-\eta \varepsilon^{-\tau}, \eta \varepsilon^{-\tau}\right)$. We have

$$
S^{\varepsilon}\left(-\eta \varepsilon^{-\tau}, \eta \varepsilon^{-\tau}\right)=\int_{-\infty}^{\infty} \Psi_{\omega, \eta}^{\varepsilon}(\zeta) d \zeta
$$


where

$$
\Psi_{\omega, \eta}^{\varepsilon}(\zeta)=1_{\left[-\eta \varepsilon^{-\tau}, \eta \varepsilon^{-\tau}\right]}(\zeta) \frac{\left|\psi\left(\varepsilon^{2} \zeta+\omega \varepsilon^{2-\tau}\right)\right|^{2}}{\left|\varepsilon^{\tau} \zeta+\omega\right|^{2 H-1}} g\left(i z_{1} \zeta\right) g\left(-i z_{2} \zeta\right) .
$$

For $\omega, \eta$ and $\zeta$ fixed we have

$$
\lim _{\varepsilon \rightarrow 0} \Psi_{\omega, \eta}^{\varepsilon}(\zeta)=\widetilde{\psi}(\omega) g\left(i z_{1} \zeta\right) g\left(-i z_{2} \zeta\right) .
$$

Moreover, observing that

$$
\left|\varepsilon^{\tau} \zeta+\omega\right|^{1-2 H} \leq \max \left\{(\omega-\eta)^{1-2 H},(\omega+\eta)^{1-2 H}\right\}
$$

for every $\zeta \in\left[-\eta \varepsilon^{-\tau}, \eta \varepsilon^{-\tau}\right]$, we get

$$
\left|\Psi_{\omega, \eta}^{\varepsilon}(\zeta)\right| \leq\|\psi\|_{\infty}^{2} \max \left\{(\omega-\eta)^{1-2 H},(\omega+\eta)^{1-2 H}\right\}\left|g\left(i z_{1} \zeta\right) g\left(-i z_{2} \zeta\right)\right| .
$$

The function $\zeta \mapsto g\left(i z_{1} \zeta\right) g\left(-i z_{2} \zeta\right)$ is absolutely integrable, thus, by the bounded convergence theorem,

$$
\lim _{\varepsilon \rightarrow 0} \int_{-\infty}^{\infty} \Psi_{\omega, \eta}^{\varepsilon}(\zeta) d \zeta=\widetilde{\psi}(\omega) \int_{-\infty}^{\infty} g\left(i z_{1} \zeta\right) g\left(-i z_{2} \zeta\right) d \zeta
$$

which concludes the proof.

Lemma 4. We have the following convergence

$$
\lim _{\varepsilon \rightarrow 0} \int_{\mathbb{R}} \frac{\left|\psi\left(\varepsilon^{2} \zeta+\omega \varepsilon^{2-\tau}\right)\right|^{2}}{\left|\varepsilon^{\tau} \zeta+\omega\right|^{2 H-1}} g\left(i z_{1} \zeta\right) g\left(-i z_{2}\left(\zeta+2 \omega \varepsilon^{-\tau}\right)\right) d \zeta=0
$$

for every $z_{1}$ and $z_{2} \in \mathbb{R}$ and $\omega \neq 0$.

Proof. Without loss of generality and for simplicity of presentation we assume that $\omega=1$. We have

$$
\begin{aligned}
\int_{\mathbb{R}} \frac{\left|\psi\left(\varepsilon^{2} \zeta+\varepsilon^{2-\tau}\right)\right|^{2}}{\left|\varepsilon^{\tau} \zeta+1\right|^{2 H-1}}\left|g\left(i z_{1} \zeta\right) g\left(-i z_{2}\left(\zeta+2 \varepsilon^{-\tau}\right)\right)\right| d \zeta \\
\quad \leq\|\psi\|_{\infty}^{2} \int_{\mathbb{R}} \frac{\left|g\left(i z_{1} \zeta\right) g\left(-i z_{2}\left(\zeta+2 \varepsilon^{-\tau}\right)\right)\right|}{\left|\varepsilon^{\tau} \zeta+1\right|^{2 H-1}} d \zeta \\
\quad=\|\psi\|_{\infty}^{2} \int_{\mathbb{R}} \frac{|\exp (i z \zeta)-1|\left|\exp \left(-i z_{1}\left(\zeta+2 \varepsilon^{-\tau}\right)\right)-1\right|}{|i z \zeta|\left|-i z_{2}\left(\zeta+2 \varepsilon^{-\tau}\right)\right|\left|\varepsilon^{\tau} \zeta+1\right|^{2 H-1}} d \zeta \\
\quad=\varepsilon^{\tau} \frac{\|\psi\|_{\infty}^{2}}{\left|z_{1} z_{2}\right|} \int_{\mathbb{R}} \frac{\left|\exp \left(i z_{1} \zeta \varepsilon^{-\tau}\right)-1\right|\left|\exp \left(-i z_{2}(\zeta+2) \varepsilon^{-\tau}\right)-1\right|}{|\zeta||\zeta+2||\zeta+1|^{2 H-1}} d \zeta
\end{aligned}
$$

We next define for $a$ and $b$ in $[-\infty, \infty]$ the $\varepsilon$-dependent integral

$$
J^{\varepsilon}(a, b)=\varepsilon^{\tau} \int_{a}^{b} \frac{\left|\exp \left(i z \zeta \varepsilon^{-\tau}\right)-1\right|\left|\exp \left(-i z(\zeta+2) \varepsilon^{-\tau}\right)-1\right|}{|\zeta||\zeta+2||\zeta+1|^{2 H-1}} d \zeta .
$$

We fix a real number $0<\eta \ll 1$ sufficiently small and consider separately the integrals $J^{\varepsilon}(-\infty,-2-\eta), J^{\varepsilon}(-2-\eta,-2+\eta), J^{\varepsilon}(-2+\eta,-\eta), J^{\varepsilon}(-\eta, \eta)$ 
and $J^{\varepsilon}(\eta, \infty)$, which are defined such that

$$
\begin{aligned}
\varepsilon^{\tau} \int_{\mathbb{R}} \frac{\left|\exp \left(i z \zeta \varepsilon^{-\tau}\right)-1\right|\left|\exp \left(-i z(\zeta+2) \varepsilon^{-\tau}\right)-1\right|}{|\zeta||\zeta+2||\zeta+1|^{2 H-1}} d \zeta \\
=J^{\varepsilon}(-\infty,-2-\eta)+J^{\varepsilon}(-2-\eta,-2+\eta) \\
\quad+J^{\varepsilon}(-2+\eta,-\eta)+J^{\varepsilon}(-\eta, \eta)+J^{\varepsilon}(\eta, \infty) .
\end{aligned}
$$

Using $|\exp (i u)-1| \leq 2$ for every $u \in \mathbb{R}$, we get

$$
\begin{aligned}
& J^{\varepsilon}(-\infty,-2-\eta)+J^{\varepsilon}(-2+\eta,-\eta)+J^{\varepsilon}(\eta, \infty) \\
& \leq 4 \varepsilon^{\tau}\left(\int_{-\infty}^{-2-\eta}+\int_{-2+\eta}^{-\eta}+\int_{\eta}^{\infty}\right) \frac{d \zeta}{|\zeta||\zeta+2||\zeta+1|^{2 H-1}} .
\end{aligned}
$$

Using $|\exp (i u)-1| \leq \min (|u|, 2)$ for every $u \in \mathbb{R}$, we get

$$
\begin{aligned}
J^{\varepsilon}(-2-\eta,-2 & +\eta)+J^{\varepsilon}(-\eta, \eta) \\
& \leq 2|z|\left(\int_{-2-\eta}^{-2+\eta} \frac{d \zeta}{|\zeta||\zeta+1|^{2 H-1}}+\int_{-\eta}^{\eta} \frac{d \zeta}{|\zeta+2||\zeta+1|^{2 H-1}}\right) \\
& =\mathcal{O}(\eta) \text { (independent of } \varepsilon) .
\end{aligned}
$$

Then using (15), (16), (17) and (18) we get

$$
\lim _{\varepsilon \rightarrow 0} \int_{\mathbb{R}} \frac{\left|\psi\left(\varepsilon^{2} \zeta+\varepsilon^{2-\tau}\right)\right|^{2}}{\left|\varepsilon^{\tau} \zeta+1\right|^{2 H-1}}\left|g(i z \zeta) g\left(-i z\left(\zeta+2 \varepsilon^{-\tau}\right)\right)\right| d \zeta=0,
$$

which concludes the proof.

\section{Extension to more general periodic func- tions}

\subsection{Assumptions and results}

In this section we generalize the above results and study the asymptotic behavior of the quantity defined for every $z \geq 0$ by

$$
v_{p}^{\varepsilon}(z)=\frac{1}{\varepsilon^{\gamma}} \int_{0}^{z} \nu\left(\frac{y}{\varepsilon^{2}}\right) p\left(\frac{y}{\varepsilon^{\tau}}\right) d y
$$

where $p$ is a zero-mean periodic function. More precisely we assume that there exists $\omega>0$ and two sequences $\left\{p_{c}(k), k \geq 1\right\}$ and $\left\{p_{s}(k), k \geq 1\right\}$ such that for every $z \geq 0$

$$
p(z)=\sum_{k=1}^{\infty}\left(p_{c}(k) \cos (k \omega z)+p_{s}(k) \sin (k \omega z)\right) .
$$

We assume that the Fourier series of the right-hand side of the identity above is uniformly convergent and that the coefficients $\left\{p_{c}(k), k \geq 1\right\}$ and $\left\{p_{s}(k), k \geq 1\right\}$ satisfy

$$
\sum_{k=1}^{\infty} k^{1 / 2-H}\left(\left|p_{c}(k)\right|+\left|p_{s}(k)\right|\right)<\infty .
$$


Notice that Assumption 20 implies

$$
\sum_{k=1}^{\infty} k^{1-2 H}\left(p_{c}(k)^{2}+p_{s}(k)^{2}\right)<\infty .
$$

Theorem 2. Under the assumptions above, as $\varepsilon \rightarrow 0$ the finite dimensional distributions of $v_{p}^{\varepsilon}$ converge to those of the Brownian motion $B_{p}$ satisfying

$$
\begin{aligned}
& \mathbb{E}\left[B_{p}\left(z_{1}\right) B_{p}\left(z_{2}\right)\right] \\
& =\left\{\begin{array}{l}
\min \left\{z_{1}, z_{2}\right\} \frac{|\psi(0)|^{2}}{2|\omega|^{2 H-1}} \sum_{l=1}^{\infty} l^{1-2 H}\left(p_{c}(l)^{2}+p_{s}(l)^{2}\right) \text { if } \tau<2, \\
\min \left\{z_{1}, z_{2}\right\} \frac{|\omega|^{1-2 H}}{2} \sum_{l=1}^{\infty} \frac{|\psi(l \omega)|^{2}}{l^{2 H-1}}\left(p_{c}(l)^{2}+p_{s}(l)^{2}\right) \text { if } \tau=2 .
\end{array}\right.
\end{aligned}
$$

Next we consider another zero-mean periodic function $\tilde{p}$ defined for every $z$ by

$$
\tilde{p}(z)=\sum_{k=1}^{\infty}\left(\tilde{p}_{c}(k) \cos (k \tilde{\omega} z)+\tilde{p}_{s}(k) \sin (k \tilde{\omega} z)\right) .
$$

where the Fourier series of the right-hand side is uniformly convergent, $\tilde{\omega}>0$ and the two sequences $\left\{\tilde{p}_{c}(k), k \geq 1\right\}$ and $\left\{\tilde{p}_{s}(k), k \geq 1\right\}$ satisfy

$$
\sum_{k=1}^{\infty} k^{1 / 2-H}\left(\left|\tilde{p}_{c}(k)\right|+\left|\tilde{p}_{s}(k)\right|\right)<\infty .
$$

From this function we define the process $v_{\tilde{p}}^{\varepsilon}$ for every $z \geq 0$ by

$$
v_{\tilde{p}}^{\varepsilon}(z)=\frac{1}{\varepsilon^{\gamma}} \int_{0}^{z} \nu\left(\frac{y}{\varepsilon^{2}}\right) \tilde{p}\left(\frac{y}{\varepsilon^{\tau}}\right) d y
$$

By applying Theorem 2 we get that the finite dimensional distributions of $v_{\tilde{p}}^{\varepsilon}$ converge to those of a Brownian motion. Now we address the question of the asymptotic correlation or decorrelation of the processes $v_{p}^{\varepsilon}$ and $v_{\tilde{p}}^{\varepsilon}$.

Theorem 3. Let $z_{1}$ and $z_{2}$ be two positive real number. Under the assumptions above, as $\varepsilon \rightarrow 0$ the covariance $\mathbb{E}\left[v_{p}^{\varepsilon}\left(z_{1}\right) v_{\tilde{p}}^{\varepsilon}\left(z_{2}\right)\right]$ converges to $\mathcal{C}\left(z_{1}, z_{2}\right)$ defined as

$$
\begin{aligned}
& \mathcal{C}\left(z_{1}, z_{2}\right) \\
& =\left\{\begin{array}{c}
\min \left\{z_{1}, z_{2}\right\} \frac{|\psi(0)|^{2}}{2|n \omega|^{2 H-1}} \sum_{l=1}^{\infty} l^{1-2 H}\left(p_{c}(n l) \tilde{p}_{c}(m l)+p_{s}(n l) \tilde{p}_{s}(m l)\right) \\
\text { if } \tilde{\omega} / \omega=n / m \in \mathbb{Q} \text { with } \operatorname{gcd}(n, m)=1 \text { and } \tau<2, \\
\min \left\{z_{1}, z_{2}\right\} \frac{|n \omega|^{1-2 H}}{2} \sum_{l=1}^{\infty} \frac{|\psi(n l \omega)|^{2}}{l^{2 H-1}}\left(p_{c}(n l) \tilde{p}_{c}(m l)+p_{s}(n l) \tilde{p}_{s}(m l)\right) \\
\text { if } \tilde{\omega} / \omega=n / m \in \mathbb{Q} \text { with } \operatorname{gcd}(n, m)=1 \text { and } \tau=2, \\
0 \text { if } \tilde{\omega} / \omega \notin \mathbb{Q} .
\end{array}\right.
\end{aligned}
$$




\subsection{Proof of Theorems 2 and 3}

First we establish a technical lemma regarding the quantities $I_{j}^{\varepsilon}\left(\omega_{1}, \omega_{2}, z_{1}, z_{2}\right)$ defined in Section 3.

Lemma 5. For every $z_{1}$ and $z_{2}$, there exists a constant $C_{I}\left(z_{1}, z_{2}\right)>0$ such that for every $j \in\{1,2,3,4\}, \varepsilon \in(0,1)$ and $\omega>0$

$$
\left|I_{j}^{\varepsilon}\left(\omega, \omega, z_{1}, z_{2}\right)\right| \leq\left(\omega^{1-2 H}+\omega^{-2 H}\right) C_{I}\left(z_{1}, z_{2}\right) .
$$

As a consequence, for every $\omega$ and $z$, there exists a constant $\tilde{C}(\omega, z)>0$ such that for every $k$ and $\varepsilon$

$$
\mathbb{E}\left[v_{c}^{k \omega, \varepsilon}(z)^{2}\right]+\mathbb{E}\left[v_{s}^{k \omega, \varepsilon}(z)^{2}\right] \leq \tilde{C}(\omega, z) k^{1-2 H}
$$

Proof. Using the notation introduced in the proof of Lemma 3 and taking $\eta=\omega / 2$ we have

$$
\begin{aligned}
& I_{1}^{\varepsilon}\left(\omega, \omega, z_{1}, z_{2}\right) \\
& \quad=\frac{z_{1} z_{2}}{4} \int_{\mathbb{R}} \frac{\left|\psi\left(\varepsilon^{2} \zeta+\omega \varepsilon^{2-\tau}\right)\right|^{2}}{\left|\varepsilon^{\tau} \zeta+\omega\right|^{2 H-1}} g\left(i z_{1} \zeta\right) g\left(-i z_{2} \zeta\right) d \zeta \\
& \quad=\frac{z_{1} z_{2}}{4}\left(S^{\varepsilon}\left(-\infty,-\frac{\omega}{2 \varepsilon^{\tau}}\right)+S^{\varepsilon}\left(-\frac{\omega}{2 \varepsilon^{\tau}}, \frac{\omega}{2 \varepsilon^{\tau}}\right)+S^{\varepsilon}\left(\frac{\omega}{2 \varepsilon^{\tau}}, \infty\right)\right) .
\end{aligned}
$$

By making the substitution $\zeta \rightarrow \zeta / \varepsilon^{\tau}$ and subsequently $\zeta \rightarrow \omega \zeta$ we obtain

$$
\begin{aligned}
\left|S^{\varepsilon}\left(-\infty,-\frac{\omega}{2 \varepsilon^{\tau}}\right)\right| & \leq 4 \varepsilon^{\tau} \frac{\|\psi\|_{\infty}^{2}}{z_{1} z_{2}} \int_{-\infty}^{-\omega / 2} \frac{d \zeta}{\zeta^{2}|\zeta+\omega|^{2 H-1}} \\
& \leq 4 \omega^{-2 H} \frac{\|\psi\|_{\infty}^{2}}{z_{1} z_{2}} \int_{-\infty}^{-1 / 2} \frac{d \zeta}{\zeta^{2}|\zeta+1|^{2 H-1}}
\end{aligned}
$$

We deal with $S^{\varepsilon}\left(\omega / 2 \varepsilon^{\tau}, \infty\right)$ in a similar way. Regarding $S^{\varepsilon}\left(-\omega / 2 \varepsilon^{\tau}, \omega / 2 \varepsilon^{\tau}\right)$ we have

$$
\left|S^{\varepsilon}\left(-\frac{\omega}{2 \varepsilon^{\tau}}, \frac{\omega}{2 \varepsilon^{\tau}}\right)\right| \leq \frac{\|\psi\|_{\infty}^{2}}{z_{1} z_{2}} \int_{-\omega / 2 \varepsilon^{\tau}}^{\omega / 2 \varepsilon^{\tau}} \frac{\left|e^{i z_{1} \zeta}-1\right|\left|e^{-i z_{2} \zeta}-1\right|}{\zeta^{2}\left|\varepsilon^{\tau} \zeta+\omega\right|^{2 H-1}} d \zeta .
$$

Moreover, we have

$$
\begin{aligned}
\left|\varepsilon^{\tau} \zeta+\omega\right|^{1-2 H} & \leq \max \left\{(\omega / 2)^{1-2 H},(3 \omega / 2)^{1-2 H}\right\} \\
& =\omega^{1-2 H} \sup \left\{(1 / 2)^{1-2 H},(3 / 2)^{1-2 H}\right\}
\end{aligned}
$$

for every $\zeta \in\left[-\omega / 2 \varepsilon^{\tau}, \omega / 2 \varepsilon^{\tau}\right]$, thus

$$
\begin{aligned}
\left|S^{\varepsilon}\left(-\frac{\omega}{2 \varepsilon^{\tau}}, \frac{\omega}{2 \varepsilon^{\tau}}\right)\right| \leq \omega^{1-2 H} \max \{ & \left.(1 / 2)^{1-2 H},(3 / 2)^{1-2 H}\right\} \frac{\|\psi\|_{\infty}^{2}}{z_{1} z_{2}} \\
& \times \int_{-\infty}^{\infty} \frac{\left|e^{i z_{1} \zeta}-1\right|\left|e^{-i z_{2} \zeta}-1\right|}{\zeta^{2}} d \zeta .
\end{aligned}
$$

Hence, the lemma is proved for $I_{1}^{\varepsilon}\left(\omega, \omega, z_{1}, z_{2}\right)$. We deal with $I_{4}^{\varepsilon}\left(\omega, \omega, z_{1}, z_{2}\right)$ in the same way. Regarding $I_{2}^{\varepsilon}\left(\omega, \omega, z_{1}, z_{2}\right)$ (and $I_{3}^{\varepsilon}\left(\omega, \omega, z_{1}, z_{2}\right)$ ) we observe that by Cauchy-Schwartz inequality we have

$$
\left|I_{2}^{\varepsilon}\left(\omega, \omega, z_{1}, z_{2}\right)\right| \leq \sqrt{I_{1}^{\varepsilon}\left(\omega, \omega, z_{1}, z_{1}\right) I_{4}^{\varepsilon}\left(\omega, \omega, z_{2}, z_{2}\right)}
$$


Thus, using the bounds for $I_{1}^{\varepsilon}\left(\omega, \omega, z_{1}, z_{2}\right)$ (and $I_{4}^{\varepsilon}\left(\omega, \omega, z_{1}, z_{2}\right)$ ) completes the proof of the first part of the lemma. To prove the second part, we let

$$
\tilde{C}(\omega, z)=8\left(\omega^{1-2 H}+\omega^{-2 H}\right) C_{I}(z, z) .
$$

The following lemma deals with a uniform bound for the second moment of $v_{p}^{\varepsilon}(z)$ for every $z$.

Lemma 6. For every $z$ and $\omega$ there exists a sequence $\left\{c_{k}(\omega, z)\right\}_{k}$ of positive numbers such that for every $k$ and $\varepsilon$

$$
\left|p_{c}(k)\right| \sqrt{\mathbb{E}\left[v_{c}^{k \omega, \varepsilon}(z)^{2}\right]}+\left|p_{s}(k)\right| \sqrt{\mathbb{E}\left[v_{s}^{k \omega, \varepsilon}(z)^{2}\right]} \leq c_{k}(\omega, z)
$$

and

$$
\sum_{k=1}^{\infty} c_{k}(\omega, z)<\infty
$$

Proof. It is a direct consequence of (20) and Lemma 5.

Proof. (Theorems 2 and 3)

We have

$$
v_{p}^{\varepsilon}(z)=\frac{1}{\varepsilon^{\gamma}} \int_{0}^{z} \nu\left(\frac{y}{\varepsilon^{2}}\right) \sum_{k=1}^{\infty}\left(p_{c}(k) \cos \left(k \omega \frac{y}{\varepsilon^{\tau}}\right)+p_{s}(k) \sin \left(k \omega \frac{y}{\varepsilon^{\tau}}\right)\right) d y .
$$

Because the convergence of the Fourier series is uniform we can change the order of the integral and the infinite sum to obtain

$$
v_{p}^{\varepsilon}(z)=\sum_{k=1}^{\infty}\left(p_{c}(k) v_{c}^{k \omega, \varepsilon}(z)+p_{s}(k) v_{s}^{k \omega, \varepsilon}(z)\right) .
$$

Moreover, using Lemma 6 we get

$$
\begin{aligned}
\left|p_{c}(k) \tilde{p}_{c}(j)\right| \mathbb{E} & {\left[\left|v_{c}^{k \omega, \varepsilon}\left(z_{1}\right) v_{c}^{j \tilde{\omega}, \varepsilon}\left(z_{2}\right)\right|\right] } \\
& \leq\left|p_{c}(k) \tilde{p}_{c}(j)\right| \sqrt{\mathbb{E}\left[v_{c}^{k \omega, \varepsilon}\left(z_{1}\right)^{2}\right] \mathbb{E}\left[v_{c}^{j \tilde{\omega}, \varepsilon}\left(z_{2}\right)^{2}\right]} \\
& \leq c_{k}\left(\omega, z_{1}\right) c_{j}\left(\tilde{\omega}, z_{2}\right)
\end{aligned}
$$

where $c_{k}\left(\omega, z_{1}\right)$ and $c_{j}\left(\tilde{\omega}, z_{2}\right)$ are defined as in Lemma 6 . Then we can write

$$
\begin{aligned}
\mathbb{E}\left[v_{p}^{\varepsilon}\left(z_{1}\right) v_{\tilde{p}}^{\varepsilon}\left(z_{2}\right)\right]= & \sum_{k=1}^{\infty} \sum_{j=1}^{\infty} p_{c}(k) \tilde{p}_{c}(j) \mathbb{E}\left[v_{c}^{k \omega, \varepsilon}\left(z_{1}\right) v_{c}^{j \tilde{\omega}, \varepsilon}\left(z_{2}\right)\right] \\
& +\sum_{k=1}^{\infty} \sum_{j=1}^{\infty} p_{c}(k) \tilde{p}_{s}(j) \mathbb{E}\left[v_{c}^{k \omega, \varepsilon}\left(z_{1}\right) v_{s}^{j \tilde{\omega}, \varepsilon}\left(z_{2}\right)\right] \\
& +\sum_{k=1}^{\infty} \sum_{j=1}^{\infty} p_{s}(k) \tilde{p}_{c}(j) \mathbb{E}\left[v_{s}^{k \omega, \varepsilon}\left(z_{1}\right) v_{c}^{j \tilde{\omega}, \varepsilon}\left(z_{2}\right)\right] \\
& +\sum_{k=1}^{\infty} \sum_{j=1}^{\infty} p_{s}(k) \tilde{p}_{s}(j) \mathbb{E}\left[v_{s}^{k \omega, \varepsilon}\left(z_{1}\right) v_{s}^{j \tilde{\omega}, \varepsilon}\left(z_{2}\right)\right] .
\end{aligned}
$$


We first deal with the convergence of the first term of the right hand side of the identity above. We know from Theorem 1 that for each $(j, k)$,

$$
\lim _{\varepsilon \rightarrow 0} \mathbb{E}\left[v_{c}^{k \omega, \varepsilon}\left(z_{1}\right) v_{c}^{j \tilde{\omega}, \varepsilon}\left(z_{2}\right)\right]=\frac{C(j \omega)}{2} \inf \left(z_{1}, z_{2}\right) \mathbb{I}_{\{j \omega=k \tilde{\omega}\}} .
$$

Combining the bounded convergence theorem, 29, 27 and Lemma 6 then implies

$$
\begin{aligned}
\lim _{\varepsilon \rightarrow 0} \sum_{k=1}^{\infty} \sum_{j=1}^{\infty} p_{c}(k) \tilde{p}_{c}(j) & \mathbb{E}\left[v_{c}^{k \omega, \varepsilon}\left(z_{1}\right) v_{c}^{j \tilde{\omega}, \varepsilon}\left(z_{2}\right)\right] \\
= & \frac{\min \left(z_{1}, z_{2}\right)}{2} \sum_{k=1}^{\infty} \sum_{j=1}^{\infty} C(j \omega)^{2} \mathbb{I}_{\{j \omega=k \tilde{\omega}\}} p_{c}(k) \tilde{p}_{c}(j) .
\end{aligned}
$$

Proceeding in the same way for the other terms of the right hand side of (28) we get

$$
\begin{aligned}
\lim _{\varepsilon \rightarrow 0} \mathbb{E}\left[v_{p}^{\varepsilon}\left(z_{1}\right) v_{\tilde{p}}^{\varepsilon}\left(z_{2}\right)\right]=\frac{\min \left(z_{1}, z_{2}\right)}{2} & \sum_{k=1}^{\infty} \sum_{j=1}^{\infty} C(j \omega)^{2} \mathbb{I}_{\{j \omega=k \tilde{\omega}\}} \\
& \times\left(p_{c}(k) \tilde{p}_{c}(j)+p_{s}(k) \tilde{p}_{s}(j)\right) .
\end{aligned}
$$

If $\tilde{\omega} / \omega \in \mathbb{R}-\mathbb{Q}$ then it is obvious that

$$
\lim _{\varepsilon \rightarrow 0} \mathbb{E}\left[v_{p}^{\varepsilon}\left(z_{1}\right) v_{\tilde{p}}^{\varepsilon}\left(z_{2}\right)\right]=0 .
$$

Let us now consider that $\tilde{\omega} / \omega \in \mathbb{Q}$. Then there exist two positive integers $m$ and $n$ such that $\operatorname{gcd}(m, n)=1$ and $\tilde{\omega} m=\omega n$. If $j \omega=k \tilde{\omega}$ then $j=k n / m$ so that there exists an integer $l$ such that $k=m l$ and $j=n l$. We then conclude

$$
\begin{aligned}
\lim _{\varepsilon \rightarrow 0} \mathbb{E} & {\left[v_{p}^{\varepsilon}\left(z_{1}\right) v_{\tilde{\tilde{p}}}^{\varepsilon}\left(z_{2}\right)\right] } \\
& =\frac{\min \left(z_{1}, z_{2}\right)}{2} \sum_{l=1}^{\infty} C(n l \omega)^{2}\left(p_{c}(m l) \tilde{p}_{c}(n l)+p_{s}(m l) \tilde{p}_{s}(n l)\right)
\end{aligned}
$$

\section{$5 \quad$ Waves in randomly layered media}

In this section we return to the discussion of applications to waves, the first motivation of our work. We consider wave propagation in randomly layered media. In the model that we consider the governing equations are the Euler equations giving conservation of moments and mass:

$$
\begin{gathered}
\rho^{\varepsilon}(z) \frac{\partial u^{\varepsilon}}{\partial t}(z, t)+\frac{\partial p^{\varepsilon}}{\partial z}(z, t)=0, \\
\frac{1}{K^{\varepsilon}(z)} \frac{\partial p^{\varepsilon}}{\partial t}(z, t)+\frac{\partial u^{\varepsilon}}{\partial z}(z, t)=0,
\end{gathered}
$$

where $t$ is the time, $z$ is the depth into the medium, $p^{\varepsilon}$ is the pressure and $u^{\varepsilon}$ the particle velocity. The medium parameters are the density $\rho^{\varepsilon}$ and the 
bulk-modulus $K^{\varepsilon}$ (reciprocal of the compressibility). We assume that $\rho^{\varepsilon}$ is a constant identically equal to one in our non-dimensionalized units and $1 / K^{\varepsilon}$ is modeled as

$$
\frac{1}{K^{\varepsilon}(z)}= \begin{cases}1+\varepsilon^{\kappa} \nu\left(\frac{z}{\varepsilon^{2}}\right) & \text { for } z \in[0, Z] \\ 1 & \text { for } z \in \mathbb{R}-[0, Z]\end{cases}
$$

where $\kappa>0$. We introduce the right- and left-going waves:

$$
A^{\varepsilon}=p^{\varepsilon}+u^{\varepsilon} \quad \text { and } \quad B^{\varepsilon}=u^{\varepsilon}-p^{\varepsilon},
$$

The boundary conditions are of the form

$$
A^{\varepsilon}(z=0, t)=f\left(t / \varepsilon^{\tau}\right) \quad \text { and } \quad B^{\varepsilon}(z=Z, t)=0,
$$

for a positive real number $\tau>0$ and a source function $f$. In order to deduce a description of the transmitted pulse, we open a window of size $\varepsilon^{\tau}$ in the neighborhood of the travel time of the homogenized medium and define the processes

$$
a^{\varepsilon}(z, s)=A^{\varepsilon}\left(z, z+\varepsilon^{\tau} s\right) \quad \text { and } \quad b^{\varepsilon}(z, s)=B^{\varepsilon}\left(z,-z+\varepsilon^{\tau} s\right) .
$$

Observe that the background or homogenized medium in our scaling has a constant speed of sound equal to unity and that the medium is matched so that in the frame introduced in (33) the pulse shape is constant if $\nu \equiv 0$ or if we consider the homogenized medium [2].

An important question is the study of the asymptotics of $a^{\varepsilon}(Z, s)$ when $\varepsilon$ goes to 0 [2]. In order to address this question we introduce next the Fourier transforms $\widehat{a}^{\varepsilon}$ and $\widehat{b}^{\varepsilon}$ of $a^{\varepsilon}$ and $b^{\varepsilon}$ respectively:

$$
\widehat{a}^{\varepsilon}(z, \omega)=\int e^{i \omega s} a^{\varepsilon}(z, s) d s \quad \text { and } \quad \widehat{b}^{\varepsilon}(z, \omega)=\int e^{i \omega s} b^{\varepsilon}(z, s) d s,
$$

that satisfy

$$
\begin{aligned}
\frac{d \widehat{a}^{\varepsilon}}{d z}=\frac{i \omega}{2 \varepsilon^{\tau-\kappa}} \nu\left(\frac{z}{\varepsilon^{2}}\right)\left(\widehat{a}^{\varepsilon}-e^{-2 i \omega z / \varepsilon^{\tau} \widehat{b}^{\varepsilon}}\right), & \widehat{a}^{\varepsilon}(0, \omega)=\widehat{f}(\omega), \\
\frac{d \widehat{b}^{\varepsilon}}{d z}=\frac{i \omega}{2 \varepsilon^{\tau-\kappa}} \nu\left(\frac{z}{\varepsilon^{2}}\right)\left(e^{2 i \omega z / \varepsilon^{\tau}} \widehat{a}^{\varepsilon}-\widehat{b}^{\varepsilon}\right), & \widehat{b}^{\varepsilon}(Z, \omega)=0 .
\end{aligned}
$$

Following $[1,2]$ we express the previous system of equations in term of the propagator $P_{\omega}^{\varepsilon}(z)$ which can be written as

$$
P_{\omega}^{\varepsilon}(z)=\left(\begin{array}{cc}
\alpha_{\omega}^{\varepsilon}(z) & \overline{\beta_{\omega}^{\varepsilon}}(z) \\
\beta_{\omega}^{\varepsilon}(z) & \overline{\alpha_{\omega}^{\varepsilon}}(z)
\end{array}\right)
$$

and which satisfies

$$
\frac{d P_{\omega}^{\varepsilon}}{d z}(z)=\frac{1}{\varepsilon^{\tau-\kappa}} \mathcal{H}_{\omega}\left(\frac{z}{\varepsilon^{\tau}}, \frac{z}{\varepsilon^{2}}\right) P_{\omega}^{\varepsilon}(z), \quad P_{\omega}^{\varepsilon}(z=0)=\left(\begin{array}{cc}
1 & 0 \\
0 & 1
\end{array}\right)
$$


with

$$
\mathcal{H}_{\omega}\left(z_{1}, z_{2}\right)=\frac{i \omega}{2} \nu\left(z_{2}\right)\left(\begin{array}{cc}
1 & -e^{-2 i \omega z_{1}} \\
e^{2 i \omega z_{1}} & -1
\end{array}\right) .
$$

Defining next the transmission coefficient $T_{\omega}^{\varepsilon}$ and the reflection coefficient $R_{\omega}^{\varepsilon}$ by

$$
T_{\omega}^{\varepsilon}(z)=\frac{1}{\overline{\alpha_{\omega}^{\varepsilon}}(z)} \quad \text { and } \quad R_{\omega}^{\varepsilon}(z)=\frac{\beta_{\omega}^{\varepsilon}(z)}{\overline{\alpha_{\omega}^{\varepsilon}}(z)},
$$

we can then write

$$
a^{\varepsilon}(Z, s)=\frac{1}{2 \pi} \int e^{-i s \omega} T_{\omega}^{\varepsilon}(Z) \widehat{f}(\omega) d \omega,
$$

and

$$
b^{\varepsilon}(0, s)=\frac{1}{2 \pi} \int e^{-i s \omega} R_{\omega}^{\varepsilon}(Z) \widehat{f}(\omega) d \omega .
$$

Hence we shall study the asymptotics of the propagator $P_{\omega}^{\varepsilon}$ in order to characterize $a^{\varepsilon}$ as $\varepsilon$ goes to 0 .

What we wish to point out here is the dramatic influence of the range parameter $H$ of $\nu$. Let us recall two cases that have already been studied in previous works. For the moment we restrict ourself to the cases of slow phases $\tau \in(0,2)$.

- The case $H=1 / 2$ or the mixing case. This corresponds to the framework studied in [1] and further developed in [2]. With $\tau \in(0,2)$ we assume $\kappa=\tau-1$ to get a non-trivial asymptotic behavior. We then have the convergence as $\varepsilon$ goes to 0

$$
a^{\varepsilon}(Z, s) \longrightarrow \widetilde{a}(Z, s):=(G * f)(s-B),
$$

where $G$ is a centered Gaussian density and $B$ a Gaussian random variable.

- The case $H>1 / 2$ or the long-range case. Results on the propagation in a long-range medium have been derived in [9]. An important property of such a model is that there exists a constant $c_{H}>0$ such that

$$
\mathbb{E}[\nu(0) \nu(z)] \sim c_{H} z^{2 H-2} .
$$

In this case, we can prove that

$$
a^{\varepsilon}(Z, s) \longrightarrow \widetilde{a}(Z, s):=f(s-B),
$$

where $B$ a Gaussian random variable.

In these two cases one of the most crucial step in the proof is the convergence of the propagator $P_{\omega}^{\varepsilon}$. This consists of studying the asymptotics of the random differential equation (34). To do so, different tools are used 
depending on whether $H=1 / 2$ or $H>1 / 2$. If $H=1 / 2$ then the diffusionapproximation and martingales techniques have been used [1,2]. If $H>1 / 2$, because of the long-range property, the martingale approach cannot be used. In $[9]$ the theory of rough paths $[6,7]$ that we describe below was used.

A motivation for this work is to find a general framework dealing with the full range of $H \in(0,1)$ and in particular to address the case $H \in(0,1 / 2)$. Based on Theorem 1 we next present our conjecture regarding $H \in(0,1 / 2)$. We consider $\nu$ defined as throughout this paper, but to fix the ideas we may assume that $\nu$ is defined by $\nu(z)=B_{H}(z+1)-B_{H}(z)$ where $H \in(0,1 / 2)$. Now we assume that

$$
\tau-\kappa=\gamma=1+(2-\tau)(1-2 H) / 2
$$

and $\kappa<1$ so that $\tau \in(0,2)$. Note that the case $\gamma=0$ corresponds to the so called strong fluctuations regime, with medium fluctuations being of order one, see [2] Recall that the propagator $P_{\omega}^{\varepsilon}$ satisfies equation (34) that we can write in the form

$$
\begin{gathered}
d P_{\omega}^{\varepsilon}(z)=\frac{i \omega}{2}\left(F_{1} P_{\omega}^{\varepsilon} \circ d v_{c}^{0, \varepsilon}(z)+F_{2} P_{\omega}^{\varepsilon} \circ d v_{c}^{2 \omega, \varepsilon}(z)+F_{3} P_{\omega}^{\varepsilon} \circ d v_{s}^{2 \omega, \varepsilon}(z)\right), \\
\text { where } F_{1}=\left(\begin{array}{cc}
1 & 0 \\
0 & -1
\end{array}\right), F_{2}=\left(\begin{array}{cc}
0 & -1 \\
1 & 0
\end{array}\right) \text { and } F_{3}=\left(\begin{array}{cc}
0 & i \\
i & 0
\end{array}\right) \text { and } \\
\qquad v_{c}^{0, \varepsilon}(z)=\frac{1}{\varepsilon^{\gamma}} \int_{0}^{z} \nu\left(\frac{y}{\varepsilon^{2}}\right) d y=\frac{\varepsilon^{\tau(1-2 H) / 2}}{\varepsilon^{2-2 H}} \int_{0}^{z} \nu\left(\frac{y}{\varepsilon^{2}}\right) d y
\end{gathered}
$$

Because of $H<1 / 2,(41)$ and the invariance principle that establishes that

$$
\lim _{\varepsilon \rightarrow 0}\left\{\frac{1}{\varepsilon^{2-2 H}} \int_{0}^{z} \nu\left(\frac{y}{\varepsilon^{2}}\right) d y\right\}_{z}={ }^{\text {distribution }} B_{H},
$$

we can then observe the convergence

$$
\lim _{\varepsilon \rightarrow 0} v_{c}^{0, \varepsilon}={ }^{\text {distribution }} 0 .
$$

Let $\Omega=\left(\omega_{1}, \cdots, \omega_{n}\right)$ be a collection of frequencies. Because of (42) and Theorem 1 , as $\varepsilon$ goes to 0 , we may expect that the propagator vector $P_{\Omega}^{\varepsilon}=\left(P_{\omega_{1}}^{\varepsilon}, \cdots, P_{\omega_{n}}^{\varepsilon}\right)$ converges to $P_{\Omega}=\left(P_{\omega_{1}}, \cdots, P_{\omega_{n}}\right)$ where the asymptotic propagator $P_{\omega}$ is solution of

$$
d P_{\omega}(z)=\frac{i C(\omega) \omega}{2}\left(F_{2} P_{\omega} \circ d B_{c}^{\omega}(z)+F_{3} P_{\omega} \circ d B_{s}^{\omega}(z)\right)
$$

where $C(\omega)^{2}$ is defined by (10). Then we follow the same procedure as in [2] pp. 180-184 or as in [4] using complex martigales to get the limit distribution written as

$$
\widetilde{a}(Z, s)=\text { distribution } \frac{1}{2 \pi} \int_{-\infty}^{\infty} \exp \left(-i \omega s-\frac{\omega^{2} C(\omega)^{2}}{4} Z\right) \widehat{f}(\omega) d \omega .
$$

Hence, we get the following conjecture. 
Congecture 1. Under the above assumptions, as $\varepsilon$ goes to $0,\left\{a^{\varepsilon}(Z, s)\right\}_{s}$ converges to the random process $\{\widetilde{a}(Z, s)\}_{s}$ that can be written as

$$
\widetilde{a}(Z, s)=\left(G_{Z} * f\right)(s),
$$

where $G_{Z}$ is such that its Fourier transform is

$$
\widehat{G_{Z}}(\omega)=\exp \left(-\frac{\pi|\psi(0)|^{2}}{4}|\omega|^{3-2 H} Z\right) .
$$

Note that this means that we in the antipersistent case have a modification in the pulse shape only, while we in the long range case have a correction in the travel time only and in the mixing case a modification in both. The technical challenge in the full proof would be the rigorous convergence of $P_{\Omega}^{\varepsilon}$ to $P_{\Omega}$ as $\varepsilon \rightarrow 0$. This could be based on the use of the theory of rough paths initiated by T. Lyons [7] and for which a good reference is [6]. To summarize, the main theorem establishes that the solution $x$ of the differential system $d x=F(x) d w$ driven by a continuous and multidimensional noise $w=\left(w_{1}, . ., w_{n}\right)$ is a continuous function of the noise $w$ for a topology based on the $q$-variations of iterated integrals of $w$ for $q$ large enough. This would imply that the convergence of $P_{\Omega}^{\varepsilon}$ could be reduced to the convergence of the noises of the form $V^{\Omega, \varepsilon}$ and their iterated integrals.

\section{References}

[1] J. F. Clouet and J. P. Fouque, Spreading of a pulse travelling in random media. Annals of Applied Probability 4 1083-1097, (1994).

[2] J.P. Fouque, J. Garnier, G. Papanicolaou, and K. Solna, Wave Propagation and Time Reversal in Randomly Layered Media, Springer (2007).

[3] J. Garnier, A multi-scaled diffusion-approximation theorem. Applications to wave propagation in random media, ESAIM Probab. Statist., Vol. 1, pp. 183-206 (1997).

[4] J. Garnier and G. Papanicolaou, Analysis of pulse propagation through an one-dimensional random medium using complex martingales, Stochastics and Dynamics, Vol. 8, pp. 127-138 (2008).

[5] J. Garnier and K. Solna, Pulse propagation in random media with longrange correlation, Multiscale Model. Simul., 7, 1302-1324, (2009).

[6] A. Lejay, An introduction to rough paths, in: Séminaire de Probabilités XXXVII, Lecture Notes in Mathematics, Springer-Verlag, (2003).

[7] T. Lyons, Differential equations driven by rough signals, Rev. Mat. Iberoamer., 14:2, 215-310, (1998).

[8] R. Marty, Asymptotic behavior of differential equations driven by periodic and random processes with slowly decaying correlations, ESAIM: Probability and Statistics, Vol. 9, (2005). 
[9] R. Marty and K. Solna, Acoustic waves in long range random media. SIAM J. Appl. Math., 69, 1065-1083, (2009).

[10] R. Marty and K. Solna, A general framework for waves in random media with long-range correlations. Annals of Applied Probability, Volume 21, Number 1, pp. 115-139 (2011).

[11] G. Samorodnitsky, M. S. Taqqu, Stable non-Gaussian random processes, Chapman and Hall, (1994). 